\title{
Injection and transport properties of fast electrons in ultraintense laser-solid interactions
}

\author{
M. Coury, ${ }^{1}$ D. C. Carroll, ${ }^{1}$ A. P. L. Robinson, ${ }^{2}$ X. H. Yuan, ${ }^{1, a)}$ C. M. Brenner, ${ }^{1,2}$ M. Burza, ${ }^{3}$ \\ R. J. Gray, ${ }^{1}$ K. L. Lancaster, ${ }^{2}$ Y. T. Li ${ }^{4}$ X. X. Lin, ${ }^{4}$ D. A. MacLellan, ${ }^{1}$ H. Powell, ${ }^{1}$ \\ M. N. Quinn, ${ }^{1}$ O. Tresca, ${ }^{1}$ C.-G. Wahlström, ${ }^{3}$ D. Neely, ${ }^{2}$ and P. McKenna ${ }^{1, b)}$ \\ ${ }^{1}$ Department of Physics, SUPA, University of Strathclyde, Glasgow G4 ONG, United Kingdom \\ ${ }^{2}$ Central Laser Facility, STFC Rutherford Appleton Laboratory, Oxfordshire OX11 0QX, United Kingdom \\ ${ }^{3}$ Department of Physics, Lund University, P.O. Box 118, S-22100 Lund, Sweden \\ ${ }^{4}$ Beijing National Laboratory of Condensed Matter Physics Institute of Physics, CAS, Beijing 100190, China
}

(Received 3 January 2013; accepted 15 March 2013; published online 5 April 2013)

Fast electron injection and transport in solid foils irradiated by sub-picosecond-duration laser pulses with peak intensity equal to $4 \times 10^{20} \mathrm{~W} / \mathrm{cm}^{2}$ is investigated experimentally and via $3 \mathrm{D}$ simulations. The simulations are performed using a hybrid-particle-in-cell (PIC) code for a range of fast electron beam injection conditions, with and without inclusion of self-generated resistive magnetic fields. The resulting fast electron beam transport properties are used in rear-surface plasma expansion calculations to compare with measurements of proton acceleration, as a function of target thickness. An injection half-angle of $\sim 50^{\circ}-70^{\circ}$ is inferred, which is significantly larger than that derived from previous experiments under similar conditions. (c) 2013 American Institute of Physics. [http://dx.doi.org/10.1063/1.4799726]

\section{INTRODUCTION}

Understanding the generation and transport of large currents of fast electrons in dense targets irradiated by high intensity laser pulses is fundamentally important to the development of the fast ignition approach to inertial confinement fusion (ICF), ${ }^{1}$ the optimization of secondary radiation sources $^{2}$ and the acceleration of ions by sheath fields. ${ }^{3}$ The initial divergence of the fast electron beam and the role of self-generated resistive magnetic fields (B-fields) at the edges of the electron beam, which act to pinch or collimate it, are particularly important to the fast ignition scheme. Whether overall electron beam collimation occurs or not is highly sensitive to the initial fast electron beam divergence angle, ${ }^{4}$ and thus the total laser energy required to achieve ignition depends strongly on the electron beam divergence. ${ }^{5}$

Although early experiments involving optically probing ionization channels in transparent insulator targets (glass) revealed jet-like fast electron beams, ${ }^{6,7}$ recent studies with metallic targets, employing diagnostics based on measurements of transition radiation and X-ray emission, have concluded that the fast electron beam divergence half-angle increases with intensity, from $\sim 17^{\circ}$ at $4 \times 10^{19} \mathrm{~W} / \mathrm{cm}^{2}$ to $\sim 27^{\circ}$ at $5 \times 10^{20} \mathrm{~W} / \mathrm{cm}^{2}$ (for picosecond laser pulses). ${ }^{8,9}$ Studies have also shown that the fast electron beam injection is also sensitive to the preplasma density scale length. ${ }^{10,11}$ Recently, Honrubia and Meyer-ter-Vehn ${ }^{5}$ concluded that in order to reproduce these measured fast electron beam divergence angles in hybrid-PIC simulations, an even larger initial (injection) half-angle of $\sim 50^{\circ}$ is required. The smaller electron beam transport divergence angle arises due to the

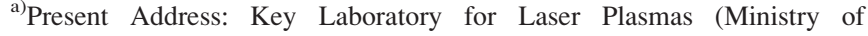
Education) and Department of Physics, Shanghai Jiao Tong University, Shanghai 200240, China.

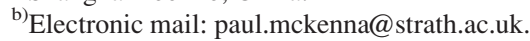

pinching effect of the self-generated resistive B-field, which is strongest in the region of the electron source where the beam current density is highest. ${ }^{4,12}$ This effect has been invoked to explain recent experimental results. ${ }^{13,14}$ The fact that the initial divergence of the fast electron beam might be large has important consequences for the fast ignition scheme and motivates further theoretical and experimental investigations of fast electron injection and transport, so that the laser pulse parameters required to achieve fast ignition can be more accurately estimated. It has also led to theoretical and experimental studies of schemes to produce collimated or focused fast electron beams. ${ }^{15-20}$

In this article, we investigate the injection and transport of fast electron beams in metallic targets driven by ultraintense $\left(I_{L}=4 \times 10^{20} \mathrm{~W} / \mathrm{cm}^{2}\right)$ picosecond laser pulses. The fast electron injection and beam transport divergence properties are inferred by measuring the maximum energies of protons and the spatial extent of fast electron-induced $K \alpha$ fluorescence and comparing the results with simulations of electron transport performed using a 3D hybrid-PIC code. We find that a large initial injection half-angle of the order of $50^{\circ}-70^{\circ}$ provides the best fit to our measurements, which is consistent with the predictions of Honrubia and Meyer-ter-Vehn. ${ }^{5}$

\section{EXPERIMENTAL DETAILS}

The experiment was performed using the Vulcan laser at the Rutherford Appleton Laboratory. Planar foils were irradiated with $p$-polarized light with wavelength, $\lambda_{L}$, equal to $1.054 \mu \mathrm{m}$, at an incident angle of $23^{\circ}$ with respect to target normal. The laser pulse duration was $\tau_{L}=(0.8 \pm 0.2) \mathrm{ps}$ and the energy (on-target) was $E_{L}=(250 \pm 25) \mathrm{J}$. The laser beam was focused by an $\mathrm{f} / 3$ off-axis parabolic mirror to an average peak-intensity of $4 \times 10^{20} \mathrm{~W} / \mathrm{cm}^{2}$. The laser intensity contrast ratio (peak to amplified spontaneous emission pedestal) 
was measured to be greater than $10^{9}$ at $1 \mathrm{~ns}$ and $10^{8}$ at tens of picoseconds, respectively, prior to the peak of the pulse. Subsequent modelling of the resulting preplasma formation showed very limited hydrodynamic expansion prior to the arrival of the peak of the pulse.

The planar, $5 \mathrm{~mm} \times 5 \mathrm{~mm}$, targets consisted of an $\mathrm{Al}$ interaction and electron propagation layer of thickness, $L$, which was varied from 100 to $500 \mu \mathrm{m}$, a buried $5 \mu \mathrm{m}$-thick $\mathrm{Cu}$ fluorescence layer, and a $1 \mu \mathrm{m}$-thick $\mathrm{Al}$ rear surface layer, designed to prevent fluorescence of the $\mathrm{Cu}$ arising from lateral spreading of the electron current on the target rear surface. ${ }^{21}$ This target composition is hereafter referred to as Al$\mathrm{Cu}-\mathrm{Al}$. To test for the influence of fast electron refluxing within the target on the fluorescence signal, a number of shots were taken with a $500 \mu \mathrm{m}$-thick $\mathrm{CH}$ layer at the target rear (i.e., $\mathrm{Al}-\mathrm{Cu}-\mathrm{CH}$ ), which significantly reduces the number of times the fast electron population that is reflected by the surface sheath fields can pass through the $\mathrm{Cu}$ layer. It was established that although refluxing can increase the total $\mathrm{Cu} K \alpha$ yield, it has a limited effect on the size of the $K \alpha$ image, in agreement with previous work reported by Quinn et al. ${ }^{22}$ The $\mathrm{CH}$ layer was not used for the main set of measurements reported below because it strongly affects rearsurface proton acceleration, and thus prevents the $K \alpha$ and proton emission diagnostic approaches being simultaneously applied.

A spherically bent Bragg crystal coupled with a FujiFilm BAS image plate detector, ${ }^{23,24}$ viewing from the target rear surface side, was used to make time-integrated 2D measurements of the $\mathrm{K} \alpha$ fluorescence of the $\mathrm{Cu}$ layer. The magnification of the imaging system was 10, giving a spatial resolution of $\sim 20 \mu \mathrm{m}$ at the source.

Dosimetry film (RCF HD-810) stacks were positioned $5 \mathrm{~cm}$ from the rear of the target and used to measure the spatial-intensity distribution of the beam of protons accelerated from the target rear surface via the target normal sheath acceleration (TNSA) mechanism. The film layers act to slow down and stop protons, and the proton beam spectrum is deconvolved in energy steps, which depend on the number and thickness of the films used. ${ }^{25}$ The stack composition used in this experiment enabled the proton energy spectrum to be measured in the range 1.2 to $40 \mathrm{MeV}$. The maximum energy measured was $(32 \pm 3) \mathrm{MeV}$.

\section{EXPERIMENT RESULTS}

The key experimental results are presented in Figure 1. Figure 1(a) shows the lateral extent (half width at half maximum, HWHM) of the $\mathrm{Cu} \mathrm{K} \alpha$ signal at two values of $L-$ the thickest target for which a clear $\mathrm{K} \alpha$ signal was recorded was $250 \mu \mathrm{m}$, which limited the thickness range over which this diagnostic approach could be applied. The scaling of the size of the $\mathrm{K} \alpha$ distribution with target thickness is in good agreement with previous measurements, reported by Lancaster et al., ${ }^{8}$ of $\mathrm{K} \alpha$ fluorescence from thinner $\mathrm{Cu}$ foils, made with the same laser and very similar laser pulse parameters, as shown in Figure 1(a). An effective fast electron beam transport half-angle of between $10^{\circ}$ and $38^{\circ}$ is inferred if a linear fit is applied to the new data points, and the corresponding
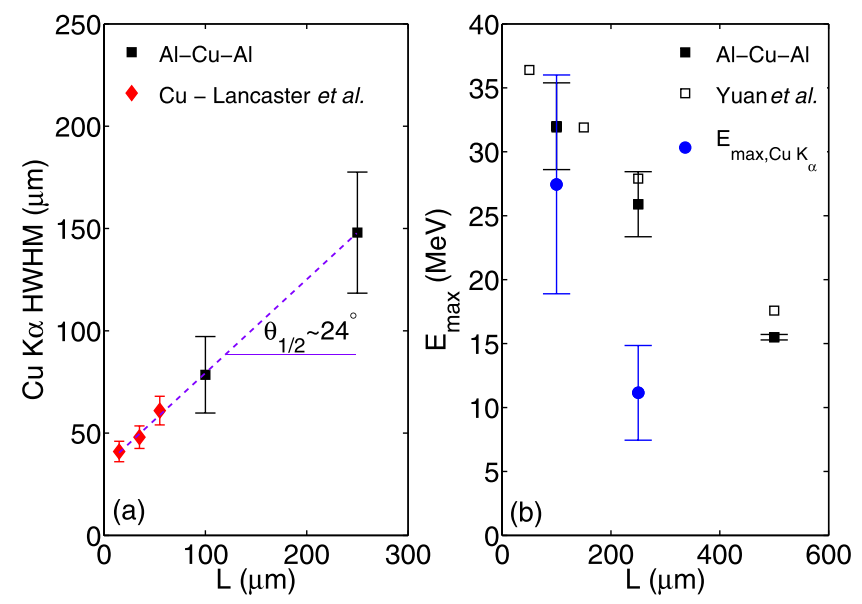

FIG. 1. (a) Measured lateral extent of $\mathrm{Cu} \mathrm{K} \alpha$ emission, half-width-at-halfmaximum (HWHM), as a function of target thickness (front $\mathrm{Al}$ transport layer), $L$. Black symbols are data from the present experiment (Al-Cu-Al). Red symbols are measurements made with similar laser pulse parameters on $\mathrm{Cu}$ targets, reproduced from Lancaster et al.; ${ }^{8}$ (b) Maximum proton energy, $E_{\text {max }}$, as a function of target thickness. Black squares are data from the present experiment $(\mathrm{Al}-\mathrm{Cu}-\mathrm{Al})$ and white squares are measurements made with similar laser pulse parameters and $\mathrm{Al}$ targets, reproduced from Yuan et al. ${ }^{14}$ Blue circles are theoretical estimates of $E_{\max }$, calculated using a plasma expansion model together with estimates of the rear-surface fast electron density inferred from the $\mathrm{Cu} \mathrm{K} \alpha$ measurements shown in (a). See main text for details.

limits are $17^{\circ}$ and $31^{\circ}$ if all data points shown are included. The best fit is for half-angle equal to $\sim 24^{\circ}$.

Figure 1(b) shows the measured maximum proton energy, $E_{\text {max }}$, as a function of $L$. Good agreement is found with results from a previous experimental campaign involving very similar laser pulse parameters and $\mathrm{Al}$ targets (Yuan et al. ${ }^{14}$ ), highlighting the reproducibility of the measurements.

The simultaneous measurement of $\mathrm{K} \alpha$ fluorescence, providing the lateral extent of the electron beam close to the target rear surface, and the measurements of proton acceleration, resulting from the fast electron-induced sheath field at the rear surface, enables the consistency of the two diagnostic approaches to be checked. To do this, we use the electron beam radius inferred from the $\mathrm{K} \alpha$ measurements to determine the fast electron density at the target rear surface, for model calculations of proton acceleration.

In a simplified model approach, the fast electron density is assumed to be constant over the beam volume and is estimated as $\left.N_{e} /\left(\pi r_{\text {rear }}^{2} c \tau_{L}\right)\right)$, where $r_{\text {rear }}$ is the fast electron beam radius at the target rear, $c$ is the speed of light and the number of fast electrons, $N_{e}$ is determined as $\eta_{L-e} E_{L} / k T_{e}$, where $\eta_{L-e}$ is the laser-to-fast electron energy conversion efficiency, $k$ is the Boltzmann constant, and $T_{e}$ is the fast electron beam temperature. These parameters are used, together with an estimated ion acceleration time, to calculate the expected maximum proton energy, using the Mora isothermal plasma expansion formula. ${ }^{26}$ That fluid plasma expansion model is 1D and assumes quasi-charge neutrality in the sheath field. The electron density follows an exponential distribution, and the ion expansion is governed by the hydrodynamic equations of continuity and motion. Full details of the model are given in reference: Mora. ${ }^{26}$ In our calculations, ponderomotive scaling $^{27}$ is assumed, giving a fixed $k T_{e}$ equal to $6 \mathrm{MeV}$, for a peak laser intensity equal to $4 \times 10^{20} \mathrm{~W} / \mathrm{cm}^{2}$ and wavelength 
equal to $1.054 \mu \mathrm{m}$. The ion acceleration time is selected as the laser pulse duration, based on the principle that to first order this is set by the duration of the electron bunch arriving at the target rear side. The main unknown parameter is $\eta_{L-e}$, which is expected to be in the range 0.1-0.5 for the laser pulse parameters of this study - the wide range is due to its sensitivity to the density scale length of the preformed plasma on the target front side. ${ }^{11,28,29}$ Importantly, since the laser and target front surface conditions are fixed in the present study, $\eta_{L-e}$ should remain constant for all measurements reported.

Figure 1(b) shows example calculated maximum proton energies for $L=100 \mu \mathrm{m}$ and $250 \mu \mathrm{m}$ with $\eta_{L-e}=0.5$. The absolute values are not important as these depend on the selected $\eta_{L-e}$ and the other unmeasured parameters, except to note that within the expected ranges of these parameters a good match can be found to the measured $E_{\max }$, particularly in the case of the thinner target. However, it is found that the gradient in the change of the calculated maximum proton energy with target thickness is much larger than the measured one. This apparent inconsistency in the two diagnostic approaches may arise due to the simplified assumptions applied in the model calculation or to the fact that $\mathrm{K} \alpha$ fluorescence can be induced by electrons with energies as low as tens of keV and may, therefore, not provide an accurate estimate of the lateral extent of the fast electrons contributing to ion acceleration. To investigate the underlying fast electron injection and transport physics in more detail, a program of electron transport simulations have been performed, as described below.

\section{FAST ELECTRON TRANSPORT SIMULATIONS}

Using the 3D hybrid-PIC code ZEPHYROS, ${ }^{17,18,30}$ several series of simulations were performed as a function of target thickness in the range $L=75-250 \mu \mathrm{m}$. These were carried out for different average injection angles and energy conversion efficiencies to investigate the effects of these parameters on fast electron transport. Simulations were also performed with the self-generated resistive B-field suppressed to investigate the role this field plays. The resulting fast electron beam parameters at the target rear surface were extracted to calculate the expected maximum proton energy for comparison to experiment.

\section{A. Simulation details}

The simulations were performed using a $500 \mu \mathrm{m} \times$ $500 \mu \mathrm{m} \times L \mu \mathrm{m}$ box. A lower limit of $L$ (the target thickness) equal to $75 \mu \mathrm{m}$ was set by the fact that in thinner targets, the fast electrons refluxing within the target (reflected by the sheath fields on both surfaces) reach the rear surface more than once during the laser pulse duration, which changes the electron density evolution. The upper $L$ limit is set by computational limitations. In a typical set of simulations, for selected fixed electron injection parameters, runs were performed for $L=75,100,150,200$, and $250 \mu \mathrm{m}$. Most of the simulations were performed with grid size equal to $2 \mu \mathrm{m} \times 2 \mu \mathrm{m} \times 2 \mu \mathrm{m}$. A few test simulations were performed at a higher resolution of $1 \mu \mathrm{m} \times 1 \mu \mathrm{m} \times 1 \mu \mathrm{m}$ to investigate the structure of the self-generated magnetic field.
The fast electron source input parameters were chosen to closely match the experiment parameters. The fast electron beam temperature was set equal to $6 \mathrm{MeV}$ (Ref. 27), and the electrons were injected into the solid over a pulse duration of $1 \mathrm{ps}$, in a focal spot with radius equal to $4 \mu \mathrm{m}$. The two variable electron source parameters are $\eta_{L-e}$ (and hence the number of fast electrons, $N_{e}$ ) and the angle of injection. The electron energy distribution, shown in Figure 2(a), is
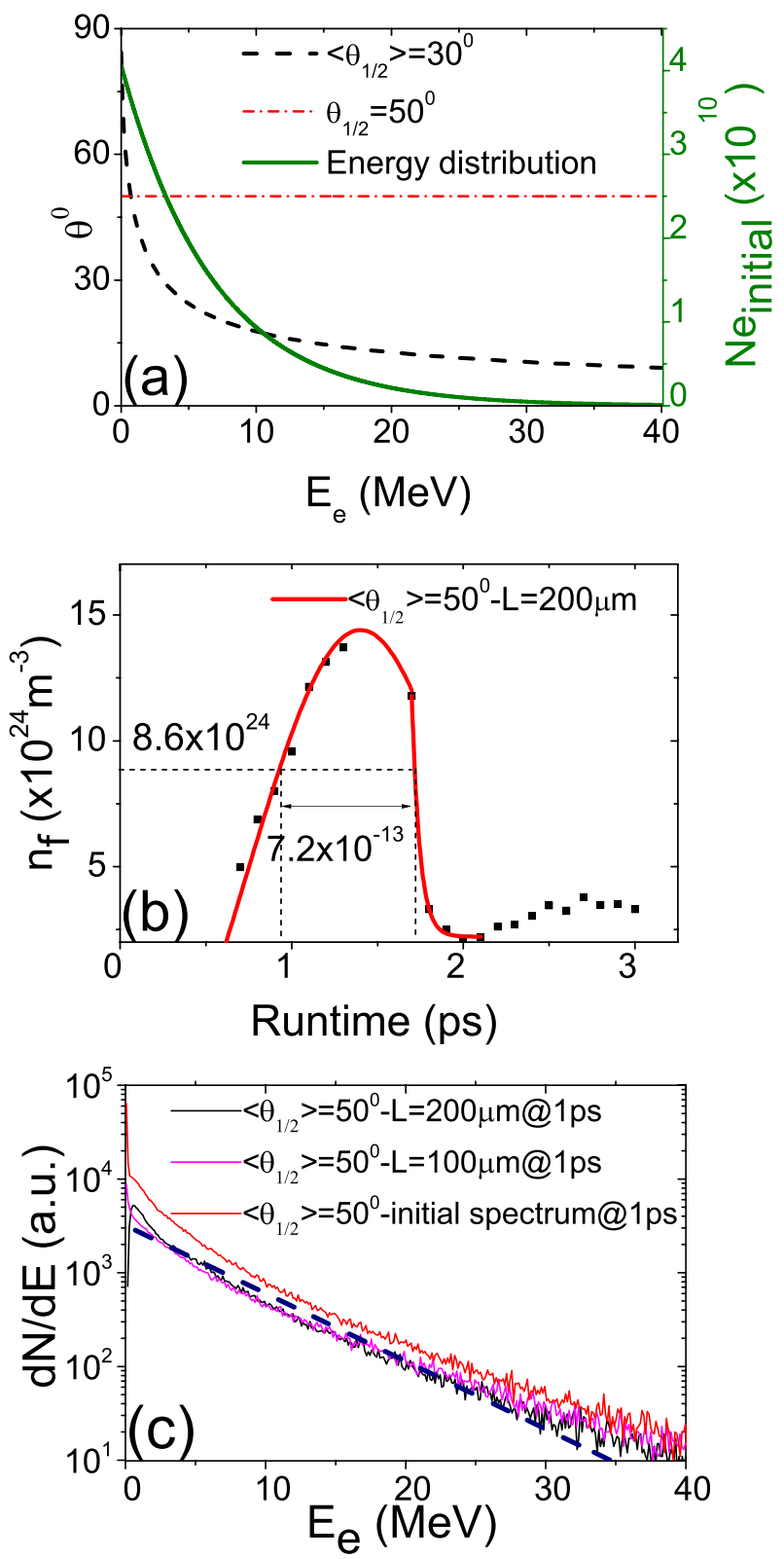

FIG. 2. (a) Fast electron beam injection half-angle as a function of electron energy. The dashed black curve is the distribution function derived by Moore et al., ${ }^{31}$ in which $\theta_{1 / 2}=\tan ^{-1}[\alpha \times \sqrt{2 /(\gamma-1)}]$, where $\gamma$ is the electron Lorentz factor and with $\alpha=1$ giving a mean half-angle $\left\langle\theta_{1 / 2}\right\rangle \sim 30^{\circ}$ in the example shown. The red line corresponds to electrons injected uniformly within a cone with half-angle equal to $50^{\circ}$ at all electron energies. The green curve is the initial fast electron energy spectrum for a beam temperature of 6 $\mathrm{MeV}$; (b) Example temporal evolution profile of the maximum fast electron density at the target rear side, extracted from a hybrid-PIC simulation of electron transport within a $200 \mu$ m-thick target, with $\left\langle\theta_{1 / 2}\right\rangle=50^{\circ}$; (c) Fast electron energy spectra extracted from the hybrid simulations at the front side ("initial spectrum") and rear side of $100 \mu \mathrm{m}$ and $200 \mu \mathrm{m}$-thick targets. The dashed line is a Boltzmann distribution with $k T_{e}=6 \mathrm{MeV}$. 
given by $N_{e}\left(E_{e}\right)=N_{e} \exp \left(-E_{e} / k T_{e}\right)$. Electrons of a given energy $E_{e}$ were uniformly injected within a cone with halfangle $\theta_{1 / 2}$, in one of two angular-energy distributions, examples of which are shown in Figure 2(a). For most of the simulation runs, the distribution function derived by Moore et al. ${ }^{31}$ was used: $\theta_{1 / 2}=\tan ^{-1}[\alpha \times \sqrt{2 /(\gamma-1)}]$, where $\gamma$ is the electron Lorentz factor and $\alpha$ is a parameter which defines the average injection angle $\left\langle\theta_{1 / 2}\right\rangle(\alpha=1$ corresponds to the angle at which electrons are ejected from the laser focal spot by the ponderomotive force). In a limited number of simulation scans, the electrons were injected uniformly into a cone with a fixed injection half-angle of $50^{\circ}$, for all $E_{e}$ (as shown in Figure 2(a)), for comparison. The model of resistivity as a function of temperature for Al used in the code is the one used previously by Davies, ${ }^{32}$ based on a fit to the data reported by Milchberg et al. $^{33}$

The peak fast electron density, $n_{f}$, and the ion acceleration time, $t_{a c c}$, as required in application of the plasma expansion formula ${ }^{26}$ to calculate $E_{\max }$ were determined from the simulation results as follows. The maximum fast electron density at the rear side of the target (usually on-axis) was plotted as a function of the simulation run time, as shown for the example in Figure 2(b). Typically, the electron density builds up over about half a picosecond to a peak value and then drops quite rapidly as fast electrons are reflected back into the target. A time-averaged value for $n_{f}$ was determined over the width of the main peak in the density-time profile, hereafter referred to as $n_{f-p e a k}$, and the FWHM duration of the peak was used for $t_{a c c}$.

In addition to $n_{f-p e a k}$ and $t_{a c c}$, the fast electron beam temperature is a third variable in the plasma expansion calculation. For the range of simulation parameters modeled, we find that $k T_{e}$ at the target rear side is largely independent of target thickness and can be approximated as the initial electron beam temperature. Figure 2(c) shows examples of the initial electron energy spectrum and the spectrum at the rear side of a $L=100 \mu \mathrm{m}$ and $L=200 \mu \mathrm{m}$ target. The target thickness affects the lowest energy, collisional electrons, but over most of the energy range the distribution is unaffected and can be approximated as a Boltzmann distribution of the form $\exp \left(-E_{e} / k T_{e}\right)$, with $k T_{e}=6 \mathrm{MeV}$ (as shown in Figure 2(c)). Hence this parameter was fixed in the plasma expansion calculations that follow.

\section{B. Role of injection angle and resistive magnetic fields in fast electron transport}

We begin with a series of simulation runs to investigate the sensitivity of fast electron beam transport to the injection angle at the source. The injection parameter $\left\langle\theta_{1 / 2}\right\rangle$ was varied from $30^{\circ}$ to $70^{\circ}$ and the resulting $n_{f-p e a k}$ and $t_{a c c}$ variations with $L$ determined. Example results are presented in Figure 3. Generally, for a given $L, n_{f-p e a k}$ decreases rapidly with increasing divergence, which is expected due to increased lateral spreading of the electrons within the target. For $\left\langle\theta_{1 / 2}\right\rangle=$ $40^{\circ}$ and above, $n_{f-\text { peak }}$ decreases with increasing $L$, also due to increased lateral spreading in the thicker targets. However, as the injection angle is decreased, the resistive azimuthal B-field produced at the edge of the fast electron beam, shown in Figure 4(a), acts over a longer beam propagation length, and in the case of $\left\langle\theta_{1 / 2}\right\rangle=30^{\circ}$ acts to pinch or collimate the electrons over the full simulation box.

The effect of the self-generated B-field is investigated by performing comparative simulations with the magnetic field growth suppressed (switched "off" in the code). As shown in the example results in Figures 5(a) and 5(b), even in the case of the relatively large $\left\langle\theta_{1 / 2}\right\rangle \sim 50^{\circ}$, the B-field strongly affects the electron density distribution within the beam and in particular the maximum electron density at a given depth in the target. The $n_{f-p e a k}$ values are significantly lower when the B-field is suppressed, irrespective of target thickness, as shown in Figure 3(a).

By comparing the fast electron density distributions (Figure 3) and the B-field distributions (Figure 4) for $\left\langle\theta_{1 / 2}\right\rangle=50^{\circ}$ and $\theta_{1 / 2}=50^{\circ}$, we note that for a given average angle of electron injection, the form of the angle-energy distribution does have some effect on the B-field generation and, therefore, the fast electron transport. However, these parameters are observed to depend much more sensitively on the magnitude of the average injection angle.

The simulation results reveal correlations between the peak electron density and the time duration over which it
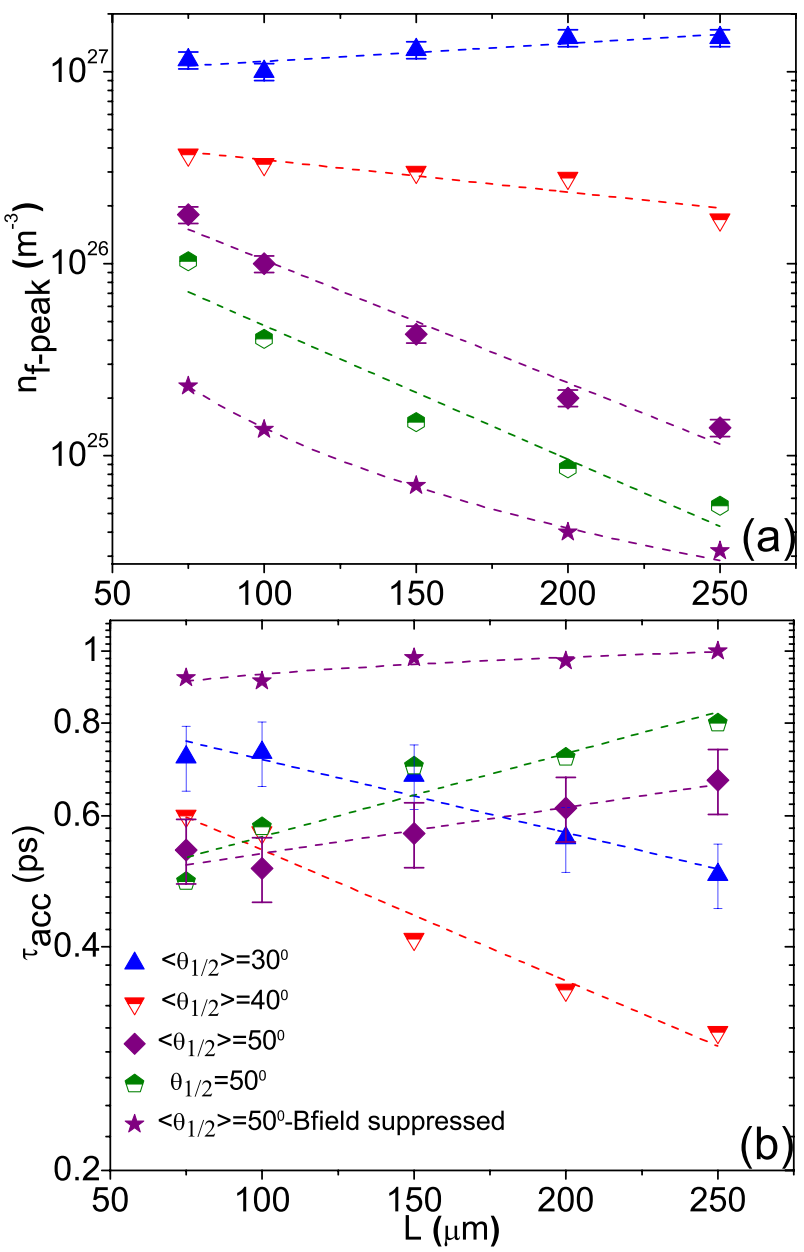

FIG. 3. (a) Fast electron density at the target rear side, averaged over the temporal peak, as a function of target thickness $L$, extracted from the hybrid simulations, for given $\left\langle\theta_{1 / 2}\right\rangle$; (b) Corresponding ion acceleration time, extracted from the FWHM temporal width of the density peak, as a function of $L$. 
evolves (and therefore, in effect, the ion acceleration time) as shown in Figure 3. Generally for low values of $\left\langle\theta_{1 / 2}\right\rangle$ for which a high $n_{f-p e a k}$ is achieved (due to the pinching effect of the B-field), the peak temporal width is small (i.e., fast rise and fall in density), whereas a lower peak density is associated with a "wider" peak and hence larger $t_{a c c}$. These correlations suggest that the resistive B-field not only affects the fast electron density distribution within the beam but also the temporal evolution of the resulting sheath field. For most of the simulation runs, $t_{a c c}$ is between 0.5 and $1.0 \mathrm{ps}$.

We note that the overall lateral extent of the fast electron beam as determined from the simulations is in good agreement with the measured extent of the $\mathrm{Cu} K \alpha$ emission for $L=100 \mu \mathrm{m}$ and $250 \mu \mathrm{m}$ within the uncertainty limits. This is demonstrated in Figure 6. However, the variation in the fast electron beam size near the target rear surface for the various different injection angles simulated is smaller than the uncertainty in the $K \alpha$ measurements, and, therefore, we are unable to draw any conclusions regarding the electron beam injection from this diagnostic. Instead, below, we look to the proton diagnostic measurements to extract this information.

\section{Calculated maximum proton energies}

As discussed above, the values of $n_{f-p e a k}$ and $t_{a c c}$ extracted from the simulation results are used as input parameters to the Mora plasma expansion model ${ }^{26}$ to obtain a
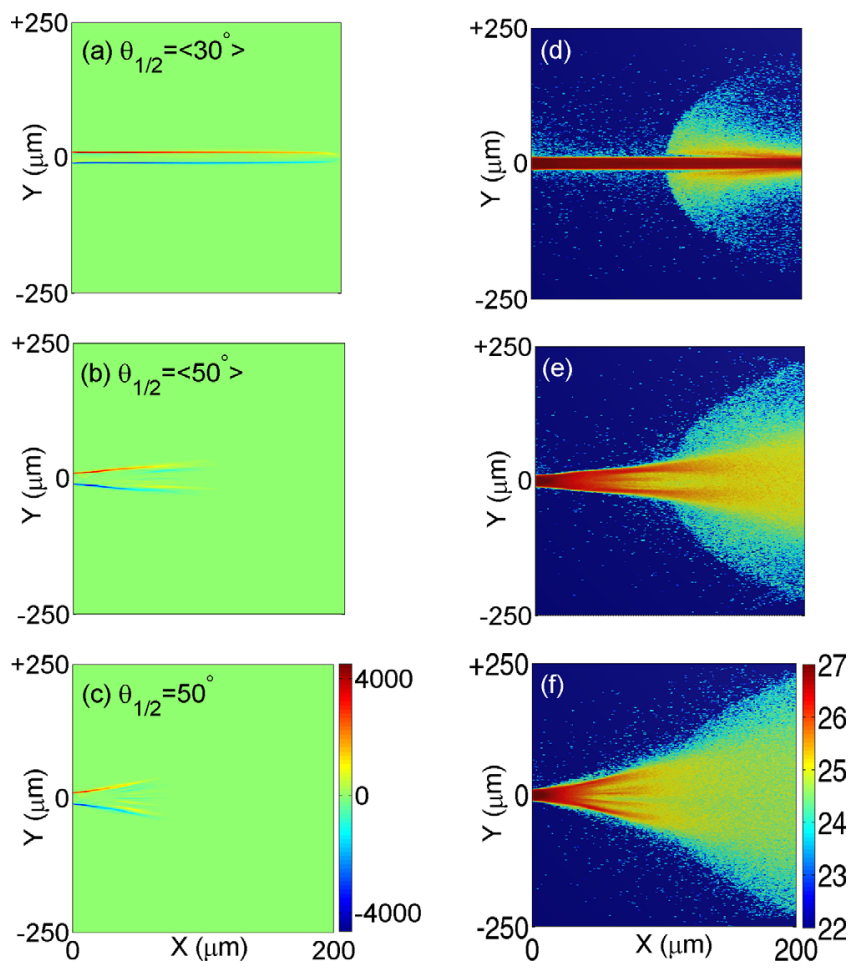

FIG. 4. ((a)-(c)) False-color 2D maps of the z-component of the selfgenerated resistive B-field (in units of Tesla); ((d)-(f)) Corresponding falsecolor $2 \mathrm{D}$ profiles of the fast electron beam density $\left(\log _{10}\left(\mathrm{~m}^{-3}\right)\right)$. Note that the $y$-axis scale is different in the two sets of plots to enable small-scale features in the resistive B-field to be viewed. The results are for a $200 \mu \mathrm{m}$-thick Al target and 1 ps runtime, at given injection half-angles specified in ((a)-(c)). The fast electrons are injected at position $(0,0,0)$ and the beam propagates in the direction of the $\mathrm{x}$-axis. The grid size for these example simulations was equal to $1 \mu \mathrm{m} \times 1 \mu \mathrm{m} \times 1 \mu \mathrm{m}$ to enable small-scale features to be resolved.

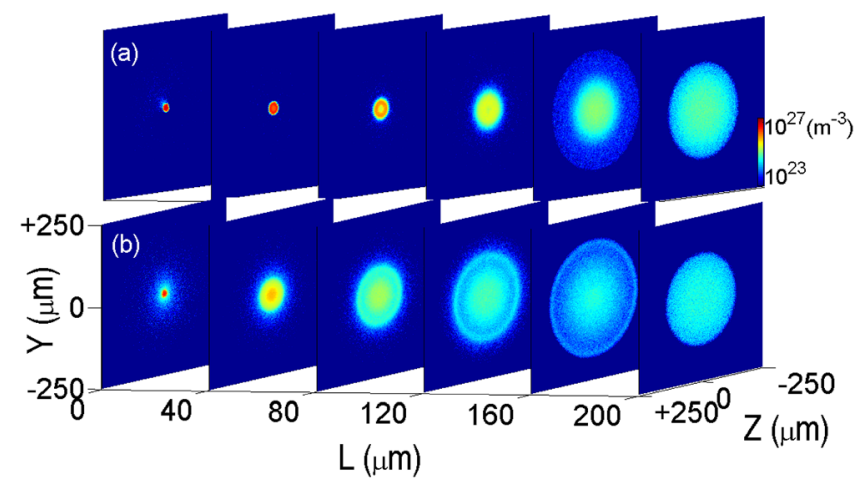

FIG. 5. (a) False-color 2D profiles of the fast electron density distribution $\left(\log _{10}\left(\mathrm{~m}^{-3}\right)\right)$ at given depths for a $200 \mu \mathrm{m}$-thick Al target for $\left\langle\theta_{1 / 2}\right\rangle \sim 50^{\circ}$ and 0.8 ps simulation time; (b) Same as (a), but with the B-field growth artificially suppressed in the simulation.

theoretical maximum proton energy for comparison to experiment. Application of this expansion formula requires that the fast electron density profile should be uniform in the plane normal to the proton motion. Although, as shown in our simulation results, the density distribution is nonuniform, the variation of the density in the region of the peak is small over a radius of the order of the Debye length, and hence the electron density can be considered to be locally uniform in the region where the fastest protons are produced.

The calculated $E_{\max }$ for a range of simulation runs are presented in Figure 7, together with the experimental results (from Figure 1(b)). For the results presented in Figure 7(a), the laser-to-electron energy conversion efficiency, $\eta_{L-e}$, is fixed at 0.2 and the average injection half-angle, $\left\langle\theta_{1 / 2}\right\rangle$, is varied. Due to the pinching effect of the B-field, the predicted proton maximum energies for the smaller injection angles is much larger than the measured $E_{\max }$. This suggests that the electrons are injected into a cone with a relatively large

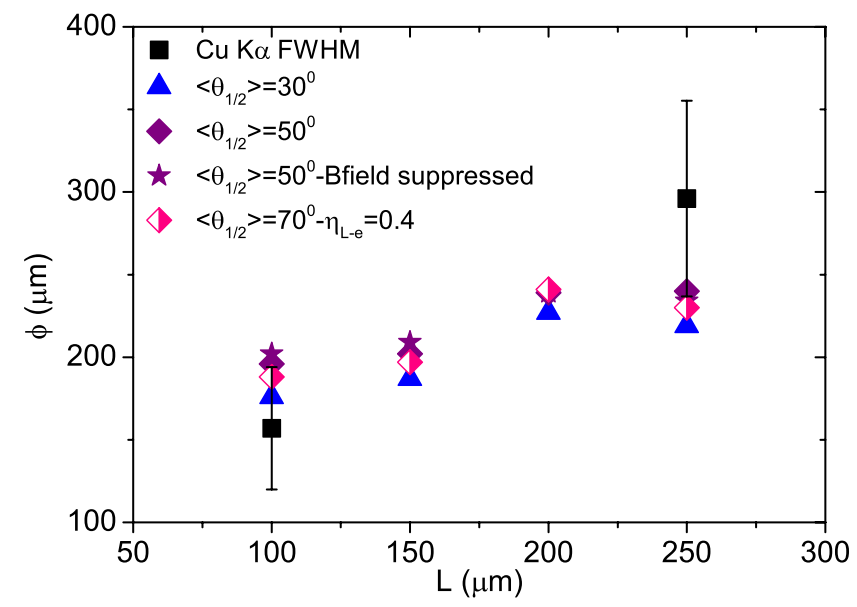

FIG. 6. Fast electron beam diameter, $\phi$, as a function of target thickness. Black squares correspond to the experimental data. Coloured symbols correspond to the lateral extent of the electron beam as determined from the simulations for given injection parameters. Unless otherwise stated, the B-field evolution is included in the simulation and $\eta_{L-e}=0.2$. The beam size is extracted from the simulation results before refluxing at the rear surface boundary at time step equal to $0.4 \mathrm{ps}$ for $L=100 \mu \mathrm{m}, 0.6 \mathrm{ps}$ for $L=150 \mu \mathrm{m}$, $0.9 \mathrm{ps}$ for $L=200 \mu \mathrm{m}$, and $1 \mathrm{ps}$ for $L=250 \mu \mathrm{m}$. 


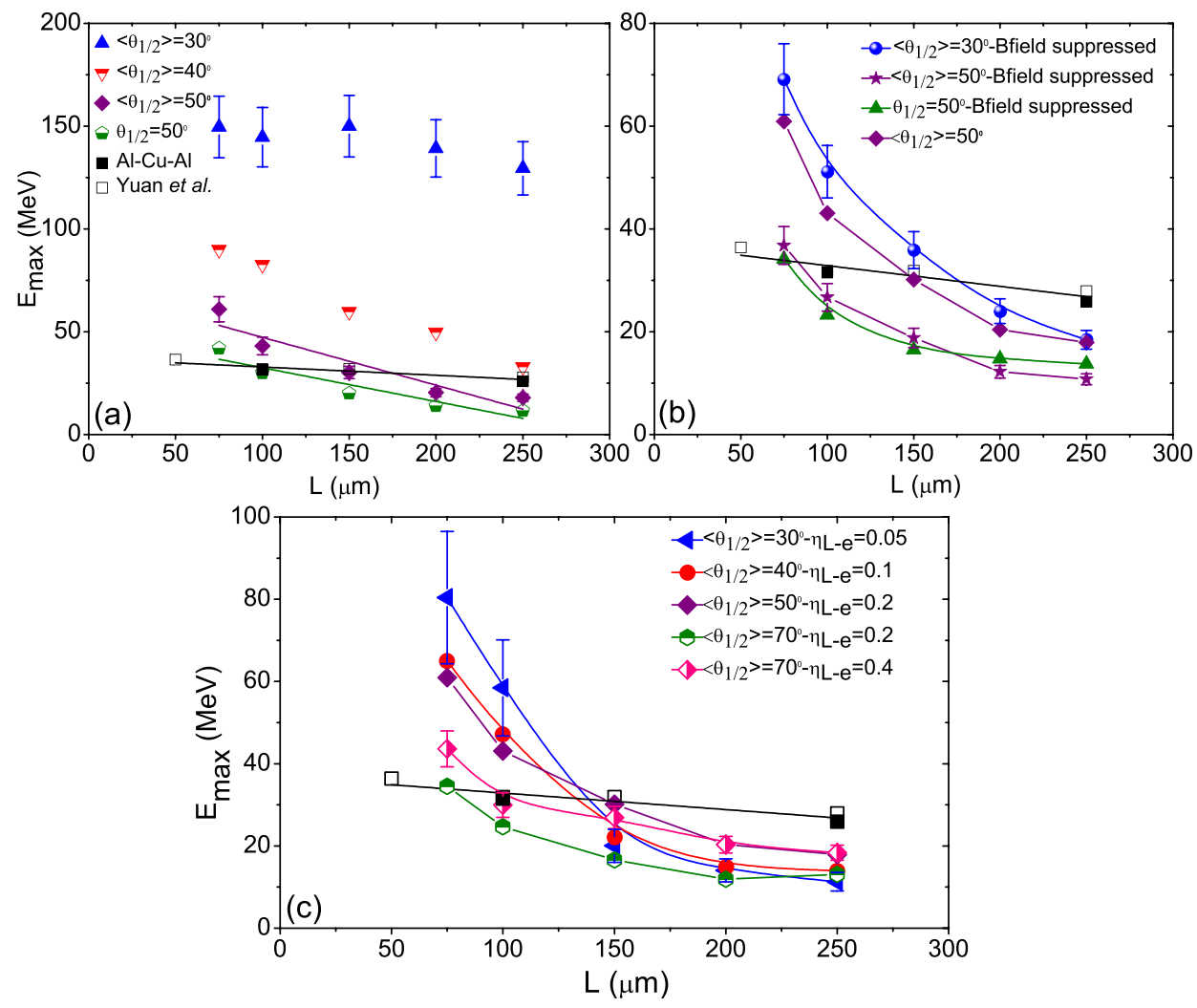

FIG. 7. Maximum proton energy as a function of $L$. As in Figure 1(b), black squares are data from the present experiment (Al-Cu-Al) and white squares are the measurements from Yuan et al. ${ }^{14}$ Colored symbols are plasma expansion model calculations of $E_{\max }$ using electron densities and ion acceleration times deduced from the hybrid simulation results of electron transport: (a) for fixed $\eta_{L-e}=0.2$ and given injection half-angles, $\left\langle\theta_{1 / 2}\right\rangle$; (b) illustrating the effect of B-field suppression (for fixed $\eta_{L-e}=0.2$ and given $\left\langle\theta_{1 / 2}\right\rangle$ ); and (c) for given $\eta_{L-e}$ and $\left\langle\theta_{1 / 2}\right\rangle$.

half-angle at $\sim 50^{\circ}$. Figure 7 (b) presents the case for which $\eta_{L-e}$ is again fixed equal to 0.2 , and the effect of suppressing the resistive $\mathrm{B}$-field is shown. In the absence of the B-field the correct order of magnitude for $E_{\text {max }}$ is obtained even for a relatively small $\left\langle\theta_{1 / 2}\right\rangle=30^{\circ}$, but the percentage decrease in $E_{\max }$ with increasing $L$ is much larger than the experimental measurement. Due to the fact that $\eta_{L-e}$ is an unknown variable which changes the value of the theoretical $E_{\text {max }}$, it is not the absolute values that should be compared, but rather the shape of the $E_{\text {max }}-L$ profile.

For the simulation results presented in Figure 7(c), both $\left\langle\theta_{1 / 2}\right\rangle$ and $\eta_{L-e}$ are varied to produce calculated proton maximum energies in the range of the experimental measurements (i.e., $\eta_{L-e}$ is increased with $\left\langle\theta_{1 / 2}\right\rangle$ to provide a better comparison to experiment). In general, we find that $\left\langle\theta_{1 / 2}\right\rangle$ must be large to ensure that realistic values of $E_{\max }$ are obtained for small $L$ (i.e., $\leq 100 \mu \mathrm{m}$ ) and that $\eta_{L-e}$ is relatively large to produce large $E_{\text {max }}$ in thick targets (i.e., $\geq 150 \mu \mathrm{m}$ ). The closest fit for the range of parameters investigated is found for $\left\langle\theta_{1 / 2}\right\rangle=70^{\circ}$ and $\eta_{L-e}=0.4$. We note that this is not an ideal fit, nor a unique solution. We have explored the variation of other possible parameters, including, for example, the injection radius of the electron beam (in the range $4-16 \mu \mathrm{m}$ ). Increasing the electron source size decreases the peak density (due to a reduction in the strength of the pinching B-field, as discussed by Bell and Kingham ${ }^{4}$ ), but increases the acceleration time (due to the inverse correlation between peak density and temporal width discussed above and shown in Figure 3), resulting in very similar maximum proton energies. We conclude on the basis of the large number of simulations conducted over a wide range of $\left\langle\theta_{1 / 2}\right\rangle$ and $\eta_{L-e}$ (and the different angle-energy injection distributions) that the average fast electron injection angle must be large to reproduce the measured $E_{\max }-L$ distribution.

\section{SUMMARY}

The injection and transport divergence properties of a high current beam of energetic electrons in metallic targets irradiated by ultraintense, picosecond laser pulses is investigated using simultaneous measurements of $\mathrm{K} \alpha$ fluorescence and proton acceleration, and a programme of 3-D hybrid-PIC simulations. The $\mathrm{K} \alpha$ fluorescence measurements, which are sensitive to the overall lateral extent of the electron beam, indicate that the effective transport half-angle is between $10^{\circ}$ and $38^{\circ}\left(17^{\circ}\right.$ and $31^{\circ}$ if previous measurements with the same laser are included) as defined by the degree of uncertainty in the measurements (best fit $\sim 24^{\circ}$ ), and this is supported by the simulation results. The simulations further reveal that the fast electron beam transport is strongly affected by self-generated magnetic fields, which in turn are sensitive to the average injection angle of the electrons at the front side of the target.

A comparison of the measured maximum proton energies with plasma expansion calculations performed using results from the electron transport simulations, indicates that the injected fast electron beam divergence at the source is significantly larger (half-angle in the range $\sim 50-70^{\circ}$ ) than inferred from previous studies performed with similar laser and target parameters. Our results support the predictions by Honrubia and Meyer-ter-Vehn ${ }^{5}$ and the recent study by Solodov et al. ${ }^{13}$ that suggest that the fast electron initial divergence angles are actually quite large. Strategies for controlling fast electron beam collimation are, therefore, likely to be important for fast ignition. 


\section{ACKNOWLEDGMENTS}

We acknowledge financial support from the EPSRC (Grant Nos. EP/E048668/1, EP/E035728/1, and EP/J003832/1) and the Swedish Research Council. We also acknowledge the support of staff at the Central Laser Facility.

${ }^{1}$ M. Tabak, J. Hammer, M. E. Glinsky, W. L. Kruer, S. C. Wilks, J. Woodworth, E. M. Campbell, M. D. Perry, and R. J. Mason, Phys. Plasmas 1, 1626-1634 (1994).

${ }^{2}$ H. Schwoerer, P. Gibbon, S. Düsterer, R. Behrens, C. Ziener, C. Reich, and R. Sauerbrey, Phys. Rev. Lett. 86, 2317-2320 (2001).

${ }^{3}$ S. C. Wilks, A. B. Langdon, T. E. Cowan, M. Roth, M. Singh, S. Hatchett, M. H. Key, D. Pennington, A. Mackinnon, and R. A. Snavely, Phys. Plasmas 8, 542 (2001)

${ }^{4}$ A. R. Bell and R. J. Kingham, Phys. Rev. Lett. 91, 035003 (2003).

${ }^{5}$ J. J. Honrubia and J. Meyer ter Vehn, Plasma Phys. Controlled Fusion 51, 014008 (2009).

${ }^{6}$ L. Gremillet, F. Amiranoff, S. D. Baton, J.-C. Gauthier, M. Koenig, E. Martinolli, F. Pisani, G. Bonnaud, C. Lebourg, C. Rousseaux, C. Toupin, A. Antonicci, D. Batani, A. Bernardinello, T. Hall, D. Scott, P. Norreys, H. Bandulet, and H. Pépin, Phys. Rev. Lett. 83, 5015-5018 (1999).

${ }^{7}$ M. Borghesi, A. J. Mackinnon, A. R. Bell, G. Malka, C. Vickers, O. Willi, J. R. Davies, A. Pukhov, and J. Meyer-ter-Vehn, Phys. Rev. Lett. 83, 4309-4312 (1999).

${ }^{8}$ K. L. Lancaster, J. S. Green, D. S. Hey, K. U. Akli, J. R. Davies, R. J. Clarke, R. R. Freeman, H. Habara, M. H. Key, R. Kodama, K. Krushelnick, C. D. Murphy, M. Nakatsutsumi, P. Simpson, R. Stephens, C. Stoeckl, T. Yabuuchi, M. Zepf, and P. A. Norreys, Phys. Rev. Lett. 98, 125002 (2007).

${ }^{9}$ J. S. Green, V. M. Ovchinnikov, R. G. Evans, K. U. Akli, H. Azechi, F. N. Beg, C. Bellei, R. R. Freeman, H. Habara, R. Heathcote, M. H. Key, J. A. King, K. L. Lancaster, N. C. Lopes, T. Ma, A. J. MacKinnon, K. Markey, A. McPhee, Z. Najmudin, P. Nilson, R. Onofrei, R. Stephens, K. Takeda, K. A. Tanaka, W. Theobald, T. Tanimoto, J. Waugh, L. Van Woerkom, N. C. Woolsey, M. Zepf, J. R. Davies, and P. A. Norreys, Phys. Rev. Lett. 100, 015003 (2008).

${ }^{10}$ V. M. Ovchinnikov, D. W. Schumacher, M. McMahon, E. A. Chowdhury, C. D. Chen, A. Morace and R. R. Freeman, Phys. Rev. Lett. 110, 065007 (2013).

${ }^{11}$ R. H. H. Scott, F. Perez, J. J. Santos, C. P. Ridgers, J. R. Davies, K. L. Lancaster, S. D. Baton, Ph. Nicolai, R. M. G. M. Trines, A. R. Bell, S. Hulin, M. Tzoufras, S. J. Rose, and P. A. Norreys, Phys. Plasmas 19, 053104 (2012).

${ }^{12}$ J. R. Davies, A. R. Bell, and M. Tatarakis, Phys. Rev. E 59, 6032 (1999).

${ }^{13}$ A. A. Solodov, M. Storm, J. F. Myatt, R. Betti, D. D. Meyerhofer, P. M. Nilson, W. Theobald, and C. Stoeckl, J. Phys.: Conf. Ser. 244, 022063 (2010).

${ }^{14}$ X. H. Yuan, A. P. L. Robinson, M. N. Quinn, D. C. Carroll, M. Borghesi, R. J. Clarke, R. G. Evans, J. Fuchs, P. Gallegos, L. Lancia, D. Neely, K. Quinn, L. Romagnani, G. Sarri, P. A. Wilson, and P. McKenna, New J. Phys. 12, 063018 (2010).
${ }^{15}$ A. P. L. Robinson and M. Sherlock, Phys. Plasmas 14, 083105 (2007).

${ }^{16}$ A. P. L. Robinson, M. Sherlock, and P. A. Norreys, Phys. Rev. Lett. 100, 025002 (2008).

${ }^{17}$ S. Kar, A. P. L. Robinson, D. C. Carroll, O. Lundh, K. Markey, P. McKenna, P. Norreys, and M. Zepf, Phys. Rev. Lett. 102, 055001 (2009).

${ }^{18}$ B. Ramakrishna, S. Kar, A. P. L. Robinson, D. J. Adams, K. Markey, M. N. Quinn, X. H. Yuan, P. McKenna, K. L. Lancaster, J. S. Green, R. H. H. Scott, P. A. Norreys, J. Schreiber, and M. Zepf, Phys. Rev. Lett. 105, 135001 (2010).

${ }^{19}$ A. P. L. Robinson, M. H. Key, and M. Tabak, Phys. Rev. Lett. 108, 125004 (2012).

${ }^{20}$ R. H. H. Scott, C. Beaucourt, H.-P. Schlenvoigt, K. Markey, K. L. Lancaster, C. P. Ridgers, C. M. Brenner, J. Pasley, R. J. Gray, I. O. Musgrave, A. P. L. Robinson, K. Li, M. M. Notley, J. R. Davies, S. D. Baton, J. J. Santos, J.-L. Feugeas, Ph. Nicolaï, G. Malka, V. T. Tikhonchuk, P. McKenna, D. Neely, S. J. Rose, and P. A. Norreys, Phys Rev. Lett. 109, 015001 (2012).

${ }^{21}$ P. McKenna, D. C. Carroll, R. J. Clarke, R. G. Evans, K. W. D. Ledingham, F. Lindau, O. Lundh, T. McCanny, D. Neely, A. P. L. Robinson, L. Robson, P. T. Simpson, C.-G. Wahlström, and M. Zepf, Phys. Rev. Lett. 98, 145001 (2007).

${ }^{22}$ M. N. Quinn, X. H. Yuan, X. X. Lin, D. C. Carroll, O. Tresca, R. J. Gray, M. Coury, C. Li, Y. T. Li, C. M. Brenner, A. P. L. Robinson, D. Neely, B. Zielbauer, B. Aurand, J. Fils, T. Kuehl, and P. McKenna, Plasma Phys. Controlled Fusion 53, 025007 (2011).

${ }^{23}$ J. A. Koch, O. L. Landen, T. W. Barbee, P. Celliers, L. B. Da Silva, S. G. Glendinning, B. A. Hammel, D. H. Kalantar, C. Brown, J. Seely, G. R. Bennett, and W. Hsing, Appl. Opt. 37, 1784-1795 (1998).

${ }^{24}$ J. A. King, K. Akli, B. Zhang, R. R. Freeman, M. H. Key, C. D. Chen, S. P. Hatchett, J. A. Koch, A. J. MacKinnon, P. K. Patel, R. Snavely, R. P. J. Town, M. Borghesi, L. Romagnani, M. Zepf, T. Cowan, H. Habara, R. Kodama, Y. Toyama, S. Karsch, K. Lancaster, C. Murphy, and P. Norreys, Appl. Phys. Lett. 86, 191501 (2005).

${ }^{25}$ F. Nürnberg, M. Schollmeier, E. Brambrink, A. Blažević, D. C. Carroll, K. Flippo, D. C. Gautier, M. Geißel, K. Harres, B. M. Hegelich, O. Lundh, K. Markey, P. McKenna, D. Neely, J. Schreiber, and M. Roth, Rev. Sci. Instrum. 80, 033301 (2009).

${ }^{26}$ P. Mora, Phys. Rev. Lett. 90, 185002 (2003).

${ }^{27}$ S. C. Wilks and W. L. Kruer, IEEE J. Quantum Electron 33, 1954-1968 (1997).

${ }^{28}$ J. R. Davies, Plasma Phys. Controlled Fusion 51, 014006 (2009).

${ }^{29}$ P. McKenna, D. C. Carroll, O. Lundh, F. Nürnberg, K. Markey, S. Bandyopadhyay, D. Batani, R. G. Evans, R. Jafer, S. Kara, D. Neely, D. Pepler, M. N. Quinn, R. Redaelli, M. Roth, C.-G. Wahlström, X. H. Yuan, and M. Zepf, Laser Part. Beams 26, 591-596 (2008).

${ }^{30}$ P. McKenna, A. P. L. Robinson, D. Neely, M. P. Desjarlais, D. C. Carroll, M. N. Quinn, X. H. Yuan, C. M. Brenner, M. Bursa, M. Coury, P. Gallegos, R. J. Gray, K. L. Lancaster, Y. T. Li, X. X. Lin, O. Tresca, and C.-G. Wahlström, Phys. Rev. Lett. 106, 185004 (2011).

${ }^{31}$ C. I. Moore, J. P. Knauer, and D. D. Meyerhofer, Phys. Rev. Lett. 74, 2439-2442 (1995).

${ }^{32}$ J. R. Davies, Phys. Rev. E 65, 026407 (2002).

${ }^{33}$ H. M. Milchberg, R. R. Freeman, S. C. Davey, and R. M. More, Phys. Rev. Lett. 61, 2364-2367 (1988). 